\title{
Weather and its effects on RSV A and B infections in infants and children in
}

\section{Korea}

\author{
Jang Mook Kim ${ }^{1}$, Jae Sik Jeon², and Jae Kyung Kim²
}

1. Department of Health Administration, Dankook University College of Health Sciences, South Korea

2. Department of Biomedical Laboratory Science, Dankook University College of Health Sciences, South

Korea

\section{RESEARCH}

Please cite this paper as: Kim JM, Jeon JS, Kim JK. Weather and its effects on RSV A and B infections in infants and children in Korea. AMJ 2017;10(12):997-1002.

https://doi.org/10.21767/AMJ.2017.3209

Corresponding Author:

Jae Kyung Kim, Ph.D.

Dankook University College of Health Sciences

Department of Biomedical Laboratory Science

119, Dandae-Ro, Dongnan-Gu, Cheonan-Si, Chungnam 31116, South Korea

Email: nerowolf2@dankook.ac.kr

\section{ABSTRACT}

\section{Background}

The epidemic patterns of respiratory syncytial virus (RSV) infection and the factors determining its seasonality are not well studied in the local society of South Korea.

\section{Aims}

This study aims to investigate the prevalence of RSV infection in hospitalized children and its relationship with climate change in Cheonan, Korea.

\section{Methods}

This retrospective study included 2,484 children (aged 0-14 years) who were hospitalized for respiratory tract infections documented RSV test at a tertiary hospital in Cheonan between December 2006 and February 2014. Daily temperature and humidity data during the period were obtained from the Korea Meteorological Administration.

\section{Results}

In total, 752 (30 per cent) showed tested positive for RSV infection, and the prevalence was noted to be higher during November and December. RSV A and RSV B were less likely to be detected simultaneously ( 0.4 per cent). RSV A was more likely to be detected during lower temperatures, lower wind speeds, and shorter daylight hours, and RSV B during lower daily precipitation and higher humidity. RSV infection was predominantly prevalent between November and April. The infection rate of RSV peaked every other year in Korea. Air temperature was associated with the epidemics of RSV infection.

\section{Conclusion}

Further understanding of the effect of climate on RSV infection will help in timely prevention using immunoprophylaxis or future vaccines.

\section{Key Words}

Climate, meteorology, respiratory syncytial virus

\section{What this study adds:}

\section{What is known about this subject?}

RSV infection can present with common cold symptoms. However, epidemiological studies of the infection route have not been conducted.

\section{What new information is offered in this study?} RSV A was more likely to be detected at lower temperatures, wind speeds, and shorter daylight hours, and RSV B during daily precipitation and higher humidity.

3. What are the implications for research, policy, or practice?

Considering the meteorological variables, we can identify the pattern of infection for RSV and provide proactive preventive guidelines for infection.

\section{Background}

Respiratory syncytial virus (RSV) is a leading cause of acute 
respiratory tract infections in infants and younger children. ${ }^{1}$ It may progress to severe pneumonia and death. ${ }^{2}$ The mortality rate associated with RSV bronchiolitis is nine times that of influenza virus during the first year of life. ${ }^{3}$ Compared with adults, infants are increased risk of complications partly related to immaturity of their immune system. ${ }^{4}$ And they are inherently sensitive to changes in weather because they are physiologically and metabolically less effective at adapting to ambient humidity and other weather-related exposures. ${ }^{5}$

In recent years, it has been increasingly recognized that ambient temperature has a significant impact on the prevalence of paediatric respiratory illnesses. ${ }^{6}$ Lower temperature, lower ultraviolet (UV) B radiation, and higher humidity prolong the survival of respiratory viruses in the environment. ${ }^{7}$ In temperate countries, seasonal variation of activity of respiratory viruses has been reported, particularly in association with temperature. ${ }^{8}$ RSV have been reported to peak during winter in both of the northern and southern hemispheres. ${ }^{9}$ RSV activity tends to rise in the coldest months in temperate regions, where winter outbreaks are common, and in the warmest months in subtropical climates. $^{10}$

Identifying geographical and seasonal distribution, weather conditions, and avoiding infection plays an important role in preventing the spread of infection. ${ }^{11}$ Therefore, we studied the pattern of RSV infections and its relationship to meteorological variations in the local city of Cheonan from 2006-2014, especially in infants less than one year of age and analysed the effect of each meteorological variable on RSV infection.

\section{Method}

The present study was approved by the institutional review board (IRB) of Dankook University (Date of IRB approval: 2015.10.13; IRB approval No.: 2015-09-009).

\section{Data collection}

Cheonan, South Korea, comprises of two districts encompassing an area of $636.3 \mathrm{~km}^{2}$ and had a population of 700,000 in 2015 . It is situated at a latitude of $36^{\circ} 48^{\prime} \mathrm{N}$ and demonstrates a typical subtropical climate with an average annual temperature of $11.8^{\circ} \mathrm{C}$. Weather data, such as mean temperature, dew point, relative humidity, precipitation, and pressure were obtained from the Korea Meteorological Administration that used the Automatic Weather Observation System to record them between December 2006 and February 2014 in Cheonan area. A total of 6,279 patients under one years of age who were treated at
Dankook University Hospital for respiratory symptoms in the same period were included in the study. Nasopharyngeal aspirate samples for RSV PCR were collected using a mucus extractor and samples were stored at $4^{\circ} \mathrm{C}$ until nucleic acid extraction was performed.

\section{Statistical analysis}

Statistical program $\mathrm{R}$ was used to examine the relationship between meteorological factors and RSV variables. Frequency analysis was performed for individual characteristics; in this study, cross-analysis ( $x^{2}$, chi-squared) was performed to determine the relationship of weather parameters and RSV A and B, with the latter as dependent variables. Variation index factor and tolerance were used to prevent confounding factors in the regression coefficient by increasing the variance of the regression coefficient. In the study, the problem of the multicollinearity was solved using correlation coefficient. Logistic regression analysis was performed to examine the meteorological factors influencing RSV A and B infection. For each dependent variable, we assigned a value of 1 for infection and a value of 0 for no infection.

\section{Results}

\section{Analysis of patients}

A total of 6,279 patients were tested for RSV PCR during the study period. Of these, 2,484 were infants aged less than one year and 1,087 were less than 100 days old. The younger the age group, the greater was the number of patients. Of the total study population, 2,025 patients tested positive for infection with a single RSV (Table 1). And 752 patients were infected with RSV A, 500 patients with RSV $B$, and 10 patients with RSV A and B (Table 2).

RSV A and B were found to occur during October to January, and more frequently during November to December (Figure 1).

A total of 1,242 patients (50.0 per cent) were not infected with the virus, and only 10 patients ( 0.4 per cent) were infected with, both, RSV A and B (Table 2). Therefore, patients were less likely to be infected with RSV A and B simultaneously, which was statistically significant $\left(x^{2}=237.07, p<0.001\right)$.

\section{Analysis of personal and meteorological characteristics}

In the case of RSV A, there was a significant relationship between individual characteristics, except the sex of the patient. The lower the age, the higher was the likelihood of infection with it. There was a higher rate of infection with 
lower average temperate, lower average wind speed, and shorter sunshine duration in day (Table 3).

In the case of RSV B also, the lower the age, the greater was the possibility of infection. The rates of infection were higher with lower daily precipitation, higher humidity, and stronger wind speed. The statistical significance was relatively low (Table 3).

\section{Discussion}

In temperate climates, RSV activity was found to be maximal during winter. ${ }^{9,12}$ During a 10 -year study period, RSV infection was found to be prevalent in December, peaking in February, subsiding in March, April, and May, and, finally, disappearing during the summer months. ${ }^{11}$ These results suggest that RSV activity is related to temperature. ${ }^{13-15}$ and suggest that RSV activity is related to temperature. In this study, the peak RSV activity occurred from mid-October to early January. For a majority of the study period, no cases were identified between April and late September. RSV activity was also increased when the mean relative humidity was between 45-65 per cent.

The stability of RSV in aerosols is dependent on humidity. ${ }^{11}$ The maximum and minimum stability of the RSV, in large particle aerosols, for one minute were 90 per cent and less than 20 per cent humidity, respectively. In case of small particle aerosols, RSV could maintain the stable status up to 60 min at 40 per cent humidity. ${ }^{16}$ In our study, statistically significant negative correlation was found between RSV and UV radiation. These results indicating that the frequency of RSV infection was in inverse proportion to the UV radiation. UV-B was found to have a much greater effect on survival of RSV on surfaces in the environment, and, thus, had effects on transmission via contact with fomites. ${ }^{17,18}$ Previous studies have shown that the relationship between temperature, relative humidity, and frequency of occurrence can be a cause of influenza seasonality. ${ }^{19-21}$ The amplitude and timing of seasonality in the transmission rate was significantly correlated with seasonal fluctuations in potential evapotranspiration, and negatively correlated with mean vapour pressure, minimum temperature, and precipitation. $^{10}$

Our study has several limitations. First and the most important, is the variation between indoor climatic conditions and those of outdoor environments. Second, the results are limited to the Cheonan area. Third, because the study only included infants and toddlers, the results may be different from that of the whole population. Further studies are required for ascertaining these findings. However, the area has four distinct seasons because of the temperate climate. Therefore, the climate changes were clear and it was easy to observe the pattern of infection with these viruses than it would be in other areas. The findings of this study are significant because until now, RSV A and RSV B, together, have not been reported in many cases.

\section{Conclusion}

The statistical results of this study showed that the weather conditions in the winter included sunlight's UV radiation, temperature, wind speeds and humidity could increase the inactivation of influenza virus in the environment, and consequently the infection rate of RSV A and B were increased in the children population in the Cheonan, Korea. Therefore, maintaining indoor humidity at 30-40 per cent and disinfection with UV can be useful in reducing the survival of RSV within these areas. Further analyses of the environmental variables will be useful in determining the vaccination timing, patient treatment and infection prevention in the future.

\section{References}

1. Lianghua L, Yongdong Y, Bin Y, et al. Epidemiological and clinical profiles of respiratory syncytial virus infection in hospitalized neonates in Suzhou, China. BMC Infect Dis. 2015;15:431-7. doi: 10.1186/s12879-015-1155-x.

2. Rodriguez-Auad JP, Nava-Frías M, Casasola-Flores J, et al. The epidemiology and clinical characteristics of respiratory syncytial virus infection in children at a public pediatric referral hospital in Mexico. Int J Infect Dis. 2012;16:e508-13. doi: 10.1016/j.ijid.2012.03.001

3. Thompson WW, Shay DK, Weintraub E, et al. Mortality associated with influenza and respiratory syncytial virus in the United States. JAMA. 2003;289:179-86. doi: 10.1001/jama.289.2.179

4. Jinghong G, Yunzong S, Yaogui L, et al. Impact of ambient humidity on child health: A Systematic Review. PLoS One. 2014;9(12):e112508. doi: 10.1371/journal.pone.0112508

5. Balbus JM, Malina C. Identifying vulnerable subpopulations for climate change health effects in the United States. J Occup Environ Med. 2009;51:33-7. doi: 10.1097/JOM.0b013e318193e12e

6. $\mathrm{Xu} \mathrm{Z}$, Etzel RA, Su $\mathrm{H}$, et al. Impact of ambient temperature on children's health: A systematic review. Environ Res. 2012;117:120-31. doi: 10.1016/j.envres.2012.07.002.

7. Loh TP, Lai FY, Tan ES, et al. Correlations between clinical illness, respiratory virus infections and climate factors in a tropical paediatric population. Epidemiol Infect. 2011;139:1884-94. doi: 10.1017/S0950268810002955.

8. Khor CS, Sam IC, Hooi PS, et al. Epidemiology and 
seasonality of respiratory viral infections in hospitalized children in Kuala Lumpur, Malaysia: A retrospective study of 27 years. BMC Pediatr. 2012;12:32. doi: 10.1186/1471-2431-12-32.

9. Yusuf $S$, Piedimonte $G$, Auais $A$, et al. The relationship of meteorological conditions to the epidemic activity of respiratory syncytial virus. Epidemiol Infect. 2007;135:1077-90. doi: 10.1017/S095026880600776X.

10. Pitzer VE, Viboud C, Alonso WJ, et al. Environmental drivers of the spatiotemporal dynamics of respiratory syncytial virus in the United States. PLoS Pathogens. Published online: 8 January 2015. doi: 10.1371/journal.ppat.1004591.

11. Welliver R. The relationship of meteorological conditions to the epidemic activity of respiratory syncytial virus. Paediatr Respir Rev. 2009;10:6-8. doi: 10.1016/S15260542(09)70004-1.

12. Welliver RC Sr. Temperature, humidity, and ultraviolet B radiation predict community respiratory syncytial virus activity. Pediatr Infect Dis J. 2007;26:S29-35. doi: 10.1097/INF.0b013e318157da59.

13. Vandini S, Corvaglia L, Alessandroni R, et al. Respiratory syncytial virus infection in infants and correlation with meteorological factors and air pollutants. Ital J Pediatr. 2013;39:1. doi: 10.1186/1824-7288-39-1.

14. Meerhoff TJ, Paget JW, Kimpen JL, et al. Variation of respiratory syncytial virus and the relation with meteorological factors in different winter seasons. Pediatr Infect Dis J. 2009;28:860-6. doi: 10.1097/INF.0b013e3181a3e949.

15. Noyola DE, Mandeville PB. Effect of climatological factors on respiratory syncytial virus epidemics. Epidemiol Infect. 2008;136:1328-32. doi: 10.1017/S0950268807000143.

16. Rechsteiner J, Winkler KC. Inactivation of respiratory syncytial virus in aerosol. J gen Virol. 1969;5:405-10. doi: 10.1099/0022-1317-5-3-405.
17. Rodriguez-Martinez CE, Sossa-Briceño MP, AcuñaCordero R. Relationship between meteorological conditions and respiratory syncytial virus in a tropical country. Epidemiol Infect. 2015;143(12):2679-86. doi: 10.1017/S0950268814003793.

18. Boone SA, Gerba CP. Significance of fomites in the spread of respiratory and enteric viral disease. AEM. 2007;73;1687-96. doi: 10.1128/AEM.02051-06.

19. du Prel JB, Puppe W, Gröndahl B, et al. Are meteorological parameters associated with acute respiratory tract infections? Clin Infect Dis. 2009;49:8618. doi: 10.1086/605435.

20. Soebiyanto RP, Gross D, Jorgensen $P$, et al. Associations between meteorological parameters and influenza activity in Berlin (Germany), Ljubljana (Slovenia), Castile and León (Spain) and Israeli Districts. PLoS One. Published online: 26 August 2015. doi: 10.1371/journal.pone.0134701.

21. Sagripanti JL, Lytle CD. Inactivation of influenza virus by solar radiation. Photochem Photobiol. 2007;83:1278-82. doi: 10.1111/j.1751-1097.2007.00177.x.

\section{PEER REVIEW}

Not commissioned. Externally peer reviewed.

\section{CONFLICTS OF INTEREST}

The authors have no conflict of interest to declare.

\section{FUNDING}

None

\section{ETHICS COMMITTEE APPROVAL}

The present study was approved by the institutional review board (IRB) of Dankook University (Date of IRB approval: 2015.10.13; IRB approval No.: 2015-09-009). 
Figure 1: Distribution of monthly infection by year
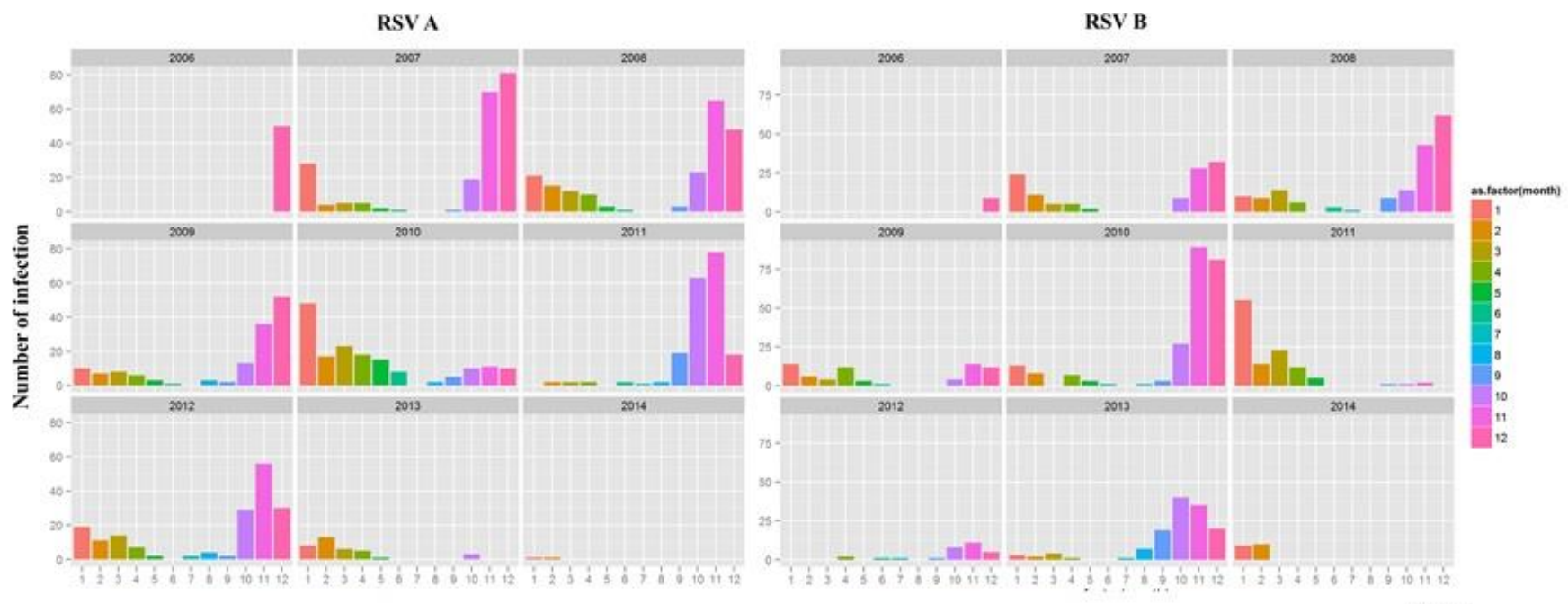

Month

Table 1: Characteristics of patients who visited Dankook University hospital

\begin{tabular}{|l|l|l|l|}
\hline Parameter & Subscripts & Specimens & (\%) \\
\hline Sex & Male & 1499 & $(60.3)$ \\
& Female & 985 & $(39.7)$ \\
\hline Age & Under 100 days & 1087 & $(43.8)$ \\
& $100-200$ days & 650 & $(26.2)$ \\
& $200-300$ days & 483 & $(19.4)$ \\
& Over 300 days & 264 & $(10.6)$ \\
\hline Multiple infection & Single & 2025 & $(81.5)$ \\
& Double & 416 & $(16.7)$ \\
& Triple & 43 & $(1.8)$ \\
\hline Infected virus & RSV A infection & 752 & $(30.3)$ \\
& RSV B infection & 500 & $(20.1)$ \\
& RSV A and B infection & 10 & $(0.4)$ \\
& Total & 2484 & $(100)$ \\
\hline
\end{tabular}

Table 2: Cross-analysis of RSV A and RSV B

\begin{tabular}{|c|c|c|c|c|c|c|c|c|}
\hline \multirow{2}{*}{\multicolumn{2}{|c|}{ Specimens (\%) }} & \multicolumn{4}{|c|}{ RSV B } & \multirow{2}{*}{\multicolumn{2}{|c|}{ Total }} & \multirow{2}{*}{$x^{2}$} \\
\hline & & \multicolumn{2}{|c|}{ Positive } & \multicolumn{2}{|c|}{ Negative } & & & \\
\hline \multirow{2}{*}{ RSV A } & Positive & 10 & $(0.4)$ & 742 & (29.9) & 752 & (30.3) & \multirow{3}{*}{$237.07^{*}$} \\
\hline & Negative & 490 & (19.7) & 1242 & (50) & 1732 & (69.7) & \\
\hline \multicolumn{2}{|l|}{ Total } & 500 & (20.1) & 1984 & (79.9) & 2484 & (100) & \\
\hline
\end{tabular}

$* P<0.001$ 
Table 3: Regression analysis results of statistically significant various variables

\begin{tabular}{|l|l|l|l|l|}
\hline \multirow{2}{*}{ Variable } & \multicolumn{2}{l}{ Association with } \\
\cline { 2 - 5 } & \multicolumn{2}{l}{ RSV A } & \multicolumn{2}{l|}{ RSV B } \\
\cline { 2 - 5 } & Estimate standard & $\boldsymbol{p}$ value & Estimate standard & $\boldsymbol{p}$ value \\
\hline Sex (male=1, female=0) & 0.084 & 0.372 & -0.019 & 0.862 \\
\hline Age & -0.003 & 0.017 & -0.002 & 0.009 \\
\hline Average temperate & -0.056 & $<0.001$ & -0.069 & $<0.001$ \\
\hline Daily precipitation & -0.013 & 0.089 & -0.045 & 0.003 \\
\hline Average wind speed & -0.263 & $<0.001$ & 0.161 & 0.01 \\
\hline Average humidity & 0.001 & 0.859 & 0.027 & $<0.001$ \\
\hline Sunshine duration in day & -0.059 & $<0.001$ & 0.017 & 0.356 \\
\hline
\end{tabular}

